\title{
Metformin induces TPC-1 cell apoptosis through endoplasmic reticulum stress-associated pathways in vitro and in vivo
}

\author{
JIANWEN YE ${ }^{1,2^{*}}$, LEI QI ${ }^{3 *}$, KUNLUN CHEN $^{1,2}$, RENFENG LI $^{1,2}$, SHENGPING SONG $^{1,2}$, \\ CHUANG ZHOU ${ }^{1,2}$ and WENLONG ZHAI ${ }^{1,2}$ \\ ${ }^{1}$ Department of Hepatobiliary and Pancreatic Surgery, The First Affiliated Hospital of Zhengzhou University; \\ ${ }^{2}$ Key Lab of Digestive Organ Transplantation of Henan Province, Open and Key Laboratory of Hepatobiliary and \\ Pancreatic Surgery and Digestive Organ Transplantation at Henan Universities, \\ Zhengzhou Key Laboratory of Hepatobiliary and Pancreatic Disease and Organ Transplantation; \\ ${ }^{3}$ Department of Pharmacy, The First Affiliated Hospital of Zhengzhou University, Zhengzhou, Henan 450052, P.R. China
}

Received September 19, 2018; Accepted May 23, 2019

DOI: 10.3892/ijo.2019.4820

\begin{abstract}
Thyroid cancer is among the most common types of malignant tumor of the endocrine system. The role of metformin in the inhibition of cancer cell proliferation and induction of apoptosis is widely accepted. The present study explored the effect and the underlying mechanisms of metformin on human thyroid cancer TPC-1 cells. Following treatment of TPC-1 cells with different concentrations of metformin, cell proliferation and apoptosis were analyzed by cell counting kit-8 (CCK-8) assay and flow cytometry, respectively. Reverse transcription-quantitative PCR and western blotting were used to detect alterations in the mRNA and protein expression levels, respectively, for heat shock protein family A member 5 (HSPA5, also known as Bip), DNA damage-inducible transcript 3 (DDIT3, also known as CHOP) and caspase-12. The results demonstrated that treatment with metformin inhibited proliferation and induced apoptosis in a concentration and time-dependent manner. In addition, treatment with metformin increased the expression of Bip, CHOP and caspase-12 in vitro, activating endoplasmic reticulum (ER) stress. Thapsigargin treatment enhanced the apoptosis induced by metformin. Inhibition of ER stress by 4-phenylbutyrate reversed the metformin-induced apoptosis. Finally, treatment with metformin inhibited thyroid cancer growth and increased the expression of Bip and CHOP in a TPC-1 cell xenograft model. These results indicated that metformin increased the apoptotic rate of thyroid cancer cells via ER stress-associated mechanisms.
\end{abstract}

Correspondence to: Dr Wenlong Zhai, Department of Hepatobiliary and Pancreatic Surgery, The First Affiliated Hospital of Zhengzhou University, 1 Jianshe East Road, Zhengzhou, Henan 450052, P.R. China E-mail: zhai-wl@hotmail.com

*Contributed equally

Key words: metformin, apoptosis, endoplasmic reticulum stress, thyroid cancer

\section{Introduction}

Thyroid cancer is among the most common types of endocrine cancer worldwide, and its incidence is increasing (1). It has been reported that thyroid cancer has the most rapidly increasing rate of incidence of all cancer types among women, and the second among men (2). Thyroid cancer can be characterized into papillary (PTC), follicular (FTC), poorly differentiated (PDTC) and anaplastic thyroid cancer (ATC) by histopathological characteristics. PTC is the most common type of thyroid cancer and accounts for $>90 \%$ of all thyroid cancer cases (3). Although multiple studies have investigated the molecular mechanisms of PTC, it remains to be fully characterized. Recent studies suggest that, as an endocrine cancer, the pathogenesis of PTC may be associated with cell metabolism, and that targeting of cancer cell metabolism may be a novel approach for the prevention or treatment of PTC (4).

Metformin, a first-line antidiabetic agent, is known to activate AMP-activated protein kinase (AMPK) and has been demonstrated to be a potential anticancer agent $(5,6)$. Epidemiologists have reported that diabetic patients who receive metformin demonstrate a lower risk and incidence of multiple types of cancer $(7,8)$. Furthermore, a number of pharmacological studies have revealed the antiproliferative and antimetastatic effects of metformin in multiple types of cancer, including thyroid cancer (9-11). Although the use of metformin decreases the incidence of cancer and induces cell death in cancer cells, its mechanism of action has not been fully investigated in thyroid cancer.

Endoplasmic reticulum (ER) stress-associated apoptosis is a major pathway in the regulation of cell apoptosis. The ER is an essential organelle for protein synthesis, folding and trafficking. A number of cellular stress conditions, such as hypoxia and metabolic stress, lead to the accumulation of nascent or unfolded proteins in the ER lumen. ER stress-induced unfolded or misfolded proteins regulate ER chaperone proteins to inhibit protein aggregation and translation and induce degradation by the proteasome; this process is termed the unfolded protein response (UPR) (12). If ER stress is persistent and unresolved, UPR triggers several signaling pathways 
leading to apoptosis. Recent studies have suggested that ER stress induces apoptosis via increasing the expression of DNA damage-inducible transcript 3 (DDIT3, also known as CHOP), JNK and caspase-12 (13). Granato et al (14) revealed that metformin enhances the cytotoxic effect of bortezomib against PEL cells by altering UPR activation and upregulating the expression of CHOP.

The present study investigated the effect of metformin on the proliferation and apoptosis of thyroid cancer TPC-1 cells, and explored the underlying molecular mechanism in vitro and in vivo. The results demonstrated that metformin inhibited cell proliferation and induced apoptosis via an ER stress-associated mechanism. The present findings suggested that metformin treatment may have therapeutic potential for patients with papillary thyroid cancer.

\section{Materials and methods}

Cell culture. The human thyroid cancer cell line, TPC-1, was obtained from the Type Culture Collection of the Chinese Academy of Sciences. The cells were cultured in RPMI-1640 (HyClone; GE Healthcare Life Sciences), supplemented with $10 \%$ fetal bovine serum (Gemini Bio Products) and antibiotics (100 U/ml penicillin and $100 \mu \mathrm{g} / \mathrm{ml}$ streptomycin), at $37^{\circ} \mathrm{C}$ in a humidified atmosphere with $5 \% \mathrm{CO}_{2}$.

Cell viability assay. Metformin, thapsigargin and 4-phenylbutyrate (4-PBA) were purchased from Sigma-Aldrich (Merck KGaA) and dissolved in dimethyl sulfoxide (DMSO). Cell viability was detected by Cell Counting kit-8 (CCK-8; Dojindo Molecular Technologies, Inc.) assay. TPC-1 cells were seeded into $96-$ well plates at a density of $5 \times 10^{3}$ cells/well. The medium was removed and replaced with medium containing metformin at $1.25,2.5,5,10$ or $20 \mathrm{mmol} / 1$ for 24 or $48 \mathrm{~h}$. Cells treated with $20 \mathrm{mmol} / 1$ metformin for $24 \mathrm{~h}$ were pretreated with $1 \mu \mathrm{mol} / 1$ thapsigargin and/or $1 \mathrm{mmol} / \mathrm{l}$ 4-phenylbutyrate for $1 \mathrm{~h}$. Then, the cells were incubated with $10 \mu \mathrm{l}$ CCK-8 solution for $1 \mathrm{~h}$. The OD values were measured by absorbance using a microplate reader (Thermo Fisher Scientific, Inc.) at a wavelength of $450 \mathrm{~nm}$, and cell viability was quantified as a percentage of the control using the OD values.

Apoptosis analysis. For cell apoptosis analysis, TPC-1 cells were analyzed using an Annexin V-FITC Apoptosis Detection kit (BD Biosciences), according to the manufacturer's instructions. Briefly, cells were collected after treatment with various concentrations of metformin for $24 \mathrm{~h}$. The cell suspension was transferred to a $5 \mathrm{ml}$ tube prior to the addition of $5 \mu \mathrm{l}$ Annexin $\mathrm{V}$ and $5 \mu \mathrm{l}$ propidium iodide (PI). After incubation in the dark at room temperature for $15 \mathrm{~min}, 400 \mu \mathrm{l}$ binding buffer was added. Apoptosis was detected with a Epics XL-MCL ADC flow cytometer (Beckman Coulter, Inc.), according to the manufacturer's instructions. Data were analyzed using the EXP032ADC operation system.

Western blot analysis. TPC-1 cells were harvested and total protein was extracted on ice using RIPA lysis buffer (Beyotime Institute of Biotechnology) with a protease inhibitor cocktail (104 mM AEBSF, $80 \mu \mathrm{M}$ aprotinin, $5 \mathrm{mM}$ bestatin, $1.5 \mathrm{mM}$ E-64, $2 \mathrm{mM}$ leupeptin, and $1.5 \mathrm{mM}$ pepstatin $\mathrm{A}$;
MedChemExpress LLC) for $20 \mathrm{~min}$. The protein concentration was determined using a bicinchoninic acid kit (Beyotime Institute of Biotechnology). Equal amounts of protein $\sim 30 \mu \mathrm{g}$ were separated by $12 \%$ SDS-PAGE and transferred into PVDF membranes (Roche Diagnostics). The membranes were immunoblotted at $4^{\circ} \mathrm{C}$ overnight, with the following primary antibodies: heat shock protein family A member 5 (HSPA5, also known as Bip; 1:800 dilution; cat. no. 11587-1-AP), CHOP (1:300 dilution; cat. no. 15204-1-AP), caspase-12 (1:500 dilution; cat. no. 55238-1-AP) and $\beta$-actin (1:5,000 dilution; cat. no. 60008-1-Ig), all from ProteinTech Group, Inc. This was followed by six 5-min washes with PBS. Then the membranes were incubated with a horseradish peroxidase-conjugated goat anti-rabbit IgG antibody (1:5,000 dilution; cat. no. JH-0011; DingGuo BioTech Co., Ltd.) for $1 \mathrm{~h}$ at room temperature, and washed six more times with PBS. The proteins were visualized using an enhanced chemiluminescence western blotting detection kit (Thermo Fisher Scientific, Inc.), according to the manufacturer's instruction. $\beta$-actin served as internal control.

Total RNA extraction and reverse transcription-quantitative PCR (RT-qPCR). Total RNA was isolated from TPC-1 cells using TRIzol (Thermo Fisher Scientific, Inc.), according to the manufacturer's instructions. cDNA was synthesized using a Reverse script RT reagent kit (Takara Biotechnology Co., Ltd.). SYBR-Green (Takara Biotechnology Co., Ltd.) was used for the qPCR to quantify the expression of Bip, caspase-12 and CHOP on the real-time PCR detection system Mx-3005P (Agilent Technologies, Inc.), according to the manufacturer's instructions. $\beta$-actin was used as a housekeeping control gene. The primer sequences used were as follows: Bip, forward, 5'-GAACGTCTG ATTGGCGATGC-3' and reverse, 5'-ACCACCTTGAACGG CAAGAA-3'; caspase-12, forward, 5'-GCTCAGGAAATGGA AACAGC-3' and reverse, 5'-AGTGCTTGGTCCCACAGATT-3'; CHOP, forward, 5'-TGGAAGCCTGGTATGAGGAC-3' and reverse, 5'-AAGCAGGGTCAAGAGTGGTG-3'; and $\beta$-actin, forward, 5'-CCTGGCACCCAGCACAAT-3' and reverse, 5'-GGGCCGGACTCGTCATAC-3'. The thermocycling conditions were: $95^{\circ} \mathrm{C}$ for $60 \mathrm{sec}$, and 40 cycles of $95^{\circ} \mathrm{C}$ for $15 \mathrm{sec}$, $60^{\circ} \mathrm{C}$ for $15 \mathrm{sec}$, and $72^{\circ} \mathrm{C}$ for $45 \mathrm{sec}$. Relative fold changes in mRNA expression were calculated using the formula $2^{-\Delta \Delta \mathrm{Cq}}(15)$.

Tumor xenograft mouse models. Ten male immunodeficient BALB/c nude mice (age, 4-6 weeks; weight, 16 $\pm 2 \mathrm{~g}$ ) were purchased from Vital River Laboratories Co., Ltd. The animals were housed under pathogen-free conditions at the Institute of Medicine Zhengzhou University at $25 \pm 2{ }^{\circ} \mathrm{C}$ and $70 \pm 5 \%$ humidity, under a 12-h light/dark cycle and access to food and water ad libitum. TPC-1 cells in the log-phase were suspended in serum-free culture medium with 50\% Matrigel(BD Biosciences) at a density of $1 \times 10^{7} \mathrm{TPC}-1$ cells in $200 \mu \mathrm{l}$. Tumor xenografts were established by subcutaneous inoculation into the right flank of nude mice. The mice were randomly divided into two groups of 5 mice: the control group, administered with PBS containing 10\% DMSO; and the metformin group, administered with $350 \mathrm{mg} / \mathrm{kg}$ intragastric metformin infusion, daily (16-18). The volume of the tumors was measured using calipers every week. Tumor volume was calculated according to the following formula: Tumor volume $\left(\mathrm{mm}^{3}\right)=\left(\right.$ length $\mathrm{x}$ width $\left.{ }^{2}\right) / 2$. The maximum tumor diameter in the present study was $15.67 \mathrm{~mm}$, 


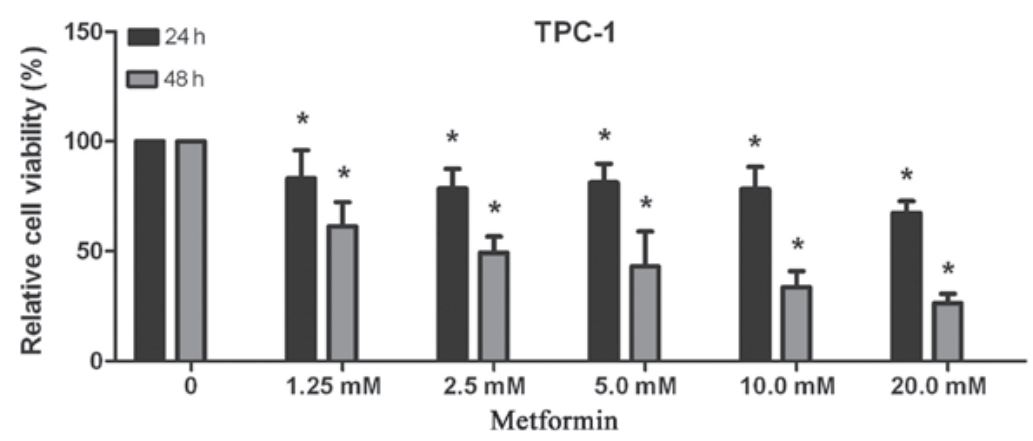

Figure 1. Metformin inhibits proliferation in TPC-1 cells. Cells were treated with the indicated concentrations of metformin for 24 and $48 \mathrm{~h}$. Cell viability was examined by Cell Counting Kit- 8 assay. ${ }^{*} \mathrm{P}<0.05$ vs. 0 mM.

A
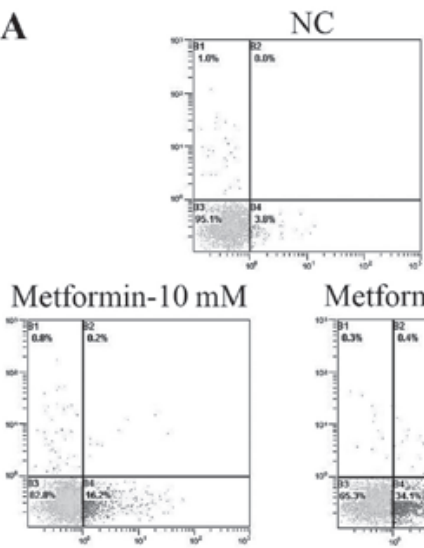

Metformin-20 mM

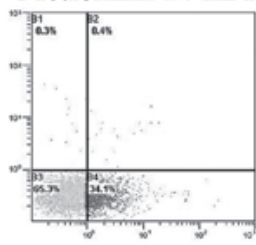

B
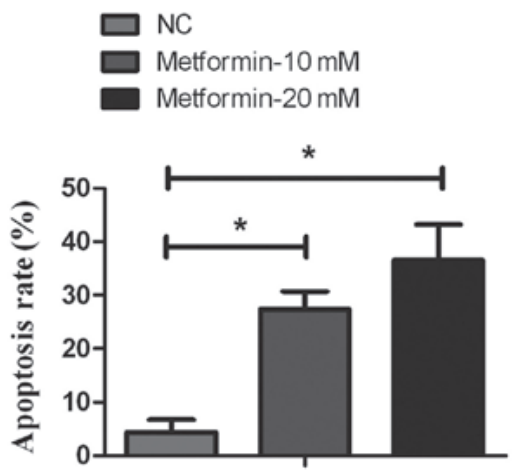

Figure 2. Metformin induces apoptosis in TPC-1 cells. TPC-1 cells were treated with 10 or $20 \mathrm{mmol} / 1 \mathrm{metformin}$ for $24 \mathrm{~h}$, stained with Annexin V-FITC and propidium iodide, then analyzed by detection by flow cytometry. (A) Representative plots. (B) Quantification of apoptosis rate. "P<0.05 vs. NC. NC, negative control

which was acceptable under the ethical guidelines. After 6 weeks, the animals were sacrificed by cervical dislocation following anesthesia by $1 \%$ pentobarbital $(50 \mathrm{mg} / \mathrm{kg})$ injection (19), and the tumor tissues were removed and measured. Xenograft tumors were harvested, fixed in $10 \%$ formalin for $24 \mathrm{~h}$ at room temperature, embedded in paraffin, and cut into $4 \mu \mathrm{m}$ sections for immunohistochemical analysis.

Immunohistochemistry. The slides were immersed in heated antigen retrieval solution (10 mmol/l citrate buffer, $\mathrm{pH} 6.0)$, and subsequently treated with $3 \%$ hydrogen peroxide for $10 \mathrm{~min}$. After washing with PBS, the slides were incubated with primary antibody (Bip, cat. no. 11587-1-AP, 1:50 dilution; CHOP, cat. no. 15204-1-AP, 1:50 dilution; both from ProteinTech Group, Inc.) at $4^{\circ} \mathrm{C}$ overnight, and then with secondary antibody (cat. no. PV9000; 1:500 dilution; OriGene Technologies, Inc.) for $20 \mathrm{~min}$ at room temperature. The reaction was developed using a 3,3'-diaminobenzidine kit (1:50 dilution in buffer; OriGene Technologies, Inc.). Finally, the slides were counterstained in hematoxylin prior to dehydration and mounting. Staining results were observed under a light microscope (CX31; Olympus Corporation; original magnification, x200). Sections were scored semi-quantitatively for the extent of immunoreaction as follows: $0,0 \%$ immunoreactive cells; $1,<5 \%$ cells; $2,5-50 \%$ immunoreactive cells; and $3,>50 \%$ immunoreactive cells. In addition, the intensity of staining was scored semi-quantitatively as: 0 , negative; 1 , weak; 2 , intermediate; and 3 , strong. The final immunoreaction score was calculated as the sum of both parameters (extent and intensity) (20).

Statistical analysis. All data are presented as the mean \pm standard deviation from at least three independent experiments. Differences between groups were assessed using Student's t-test followed by Shapiro-Wilk W test, or one-way ANOVA followed by Bonferroni test. $\mathrm{P}<0.05$ was considered to indicate a statistically significant difference, and analyses were performed using SPSS 17.0 software (SPSS Inc.).

\section{Results}

Metformin inhibits cell viability in TPC-1 cells. TPC-1 cells were seeded into 96-well plates. Following treatment with metformin at different concentrations $(1.25,2.5,5,10$ or $20 \mathrm{mmol} / \mathrm{l}$ ) for 24 or $48 \mathrm{~h}$, cell viability was detected by CCK-8 assay. As presented in Fig. 1, the inhibitory effect of metformin on proliferation increased with the increase in concentration and duration of treatment. The $\mathrm{IC}_{50}$ values of metformin for 24 and $48 \mathrm{~h}$ were 324.865 and $2.684 \mathrm{mmol} / 1$, respectively. These data indicated that metformin inhibited cell viability in a concentration- and time-dependent manner.

Metformin induces cell apoptosis in TPC-1 cells. To investigate whether the inhibition of cell proliferation was due to an increased rate of apoptosis, the proportion of apoptotic cells was detected by flow cytometry using 
A

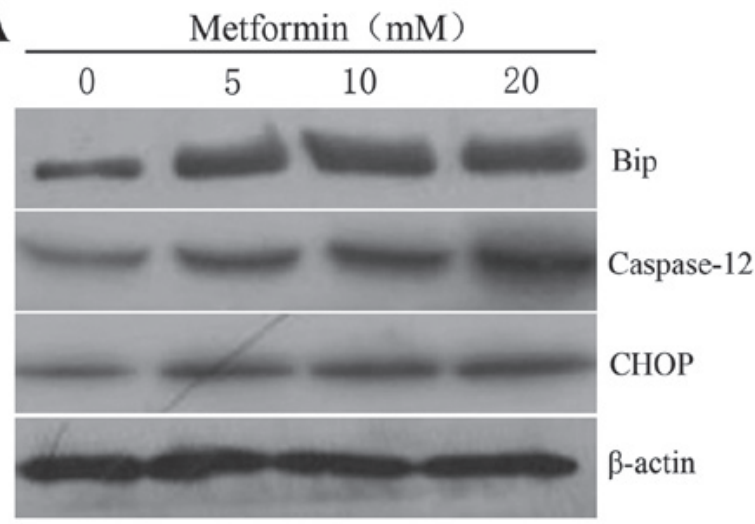

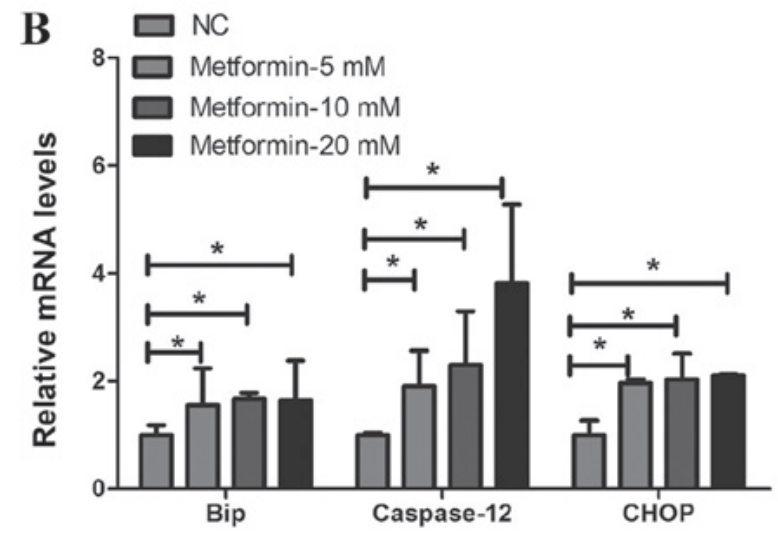

Figure 3. Metformin activates ER stress and ER stress-associated apoptosis. TPC-1 cells were treated with 5, 10 or 20 mmol/1 metformin for 24 h. (A) Protein expression levels of Bip, caspase-12 and CHOP were detected by western blotting. $\beta$-actin was used as a loading control. (B) mRNA expression levels of Bip, caspase-12 and CHOP were detected by reverse transcription-quantitative PCR. $\beta$-actin was used as an internal control. ${ }^{*} \mathrm{P}<0.05$ vs. NC. ER, endoplasmic reticulum; Bip, heat shock protein family A member 5; CHOP, DNA damage-inducible transcript 3; NC, negative control.

A

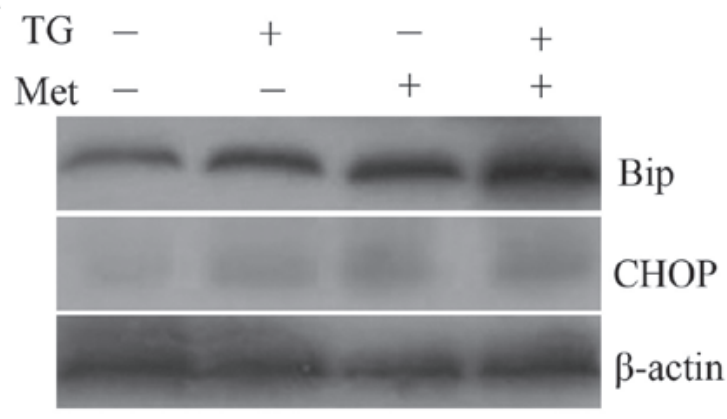

C

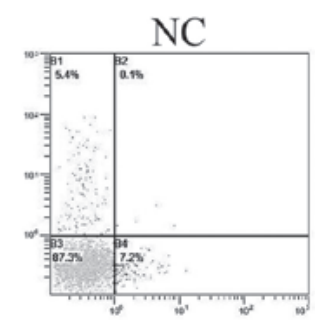

Metformin

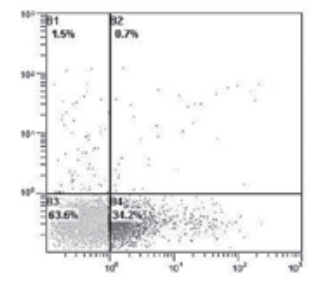

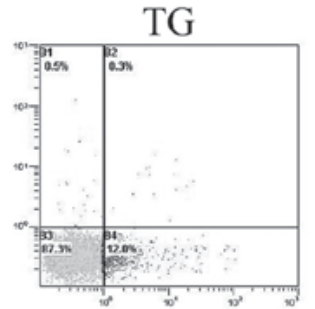

TG+Metformin

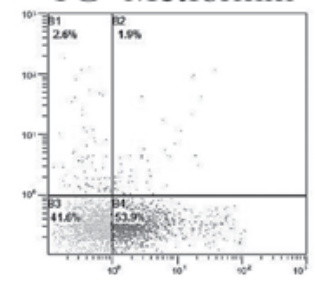

B $\square \mathrm{NC}$

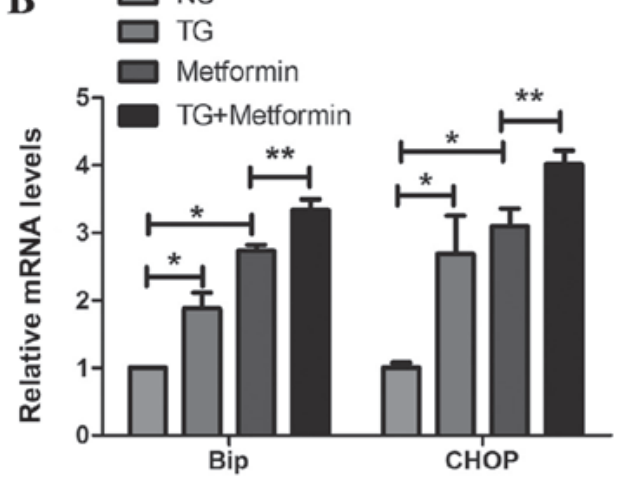

D

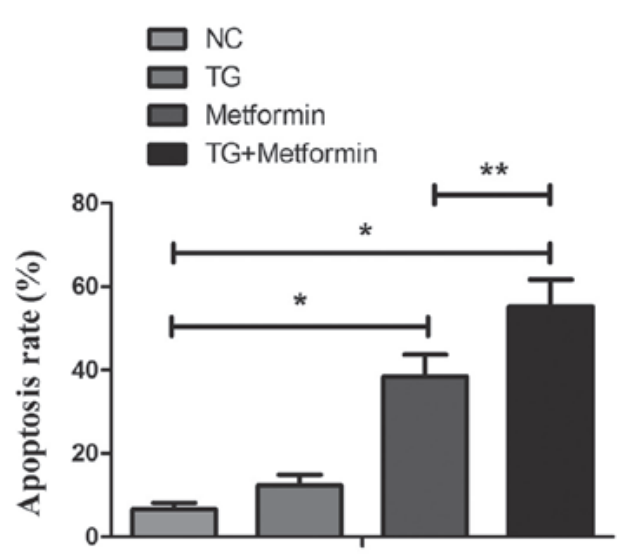

Figure 4. TG enhances metformin-mediated apoptosis in TPC-1 cells. TPC-1 cells were pretreated with TG for $1 \mathrm{~h}$, then treated with or without metformin for $24 \mathrm{~h}$. (A) The protein expression levels of Bip and CHOP were detected by western blotting, using $\beta$-actin as a loading control. (B) mRNA expression levels of Bip and CHOP following treatment with TG and/or metformin in TPC-1 cells. (C) TPC-1 cells were stained with Annexin V-FITC and propidium iodide, and analyzed flow cytometry. Representative plots are shown (D). Quantification of apoptosis rate. * $\mathrm{P}<0.05$, with comparisons indicated by brackets. TG, thapsigargin; Bip, heat shock protein family A member 5; CHOP, DNA damage-inducible transcript 3; NC, negative control; Met, metformin.

Annexin V-FITC/PI staining, following treatment with different concentrations of metformin. As presented in Fig. 2, the apoptosis rate following metformin treatment was significantly increased compared with the control group. These findings indicated that metformin induced apoptosis of thyroid cancer TPC-1 cells.
Metformin induces ER stress and ER stress-associated apoptosis in TPC-1 cells. The ER stress-associated apoptotic pathway serves an important role in apoptosis induced by anticancer agents (22). Following treatment with metformin at different concentrations for $24 \mathrm{~h}$, the mRNA and protein expression levels of the ER molecular chaperone Bip, and the 
A

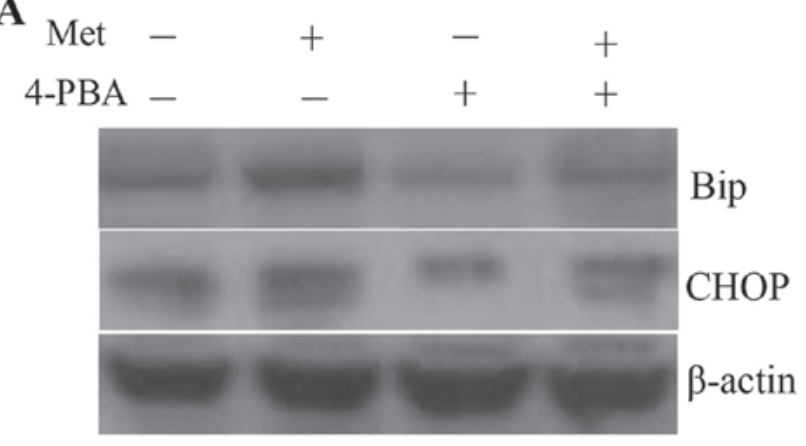

C
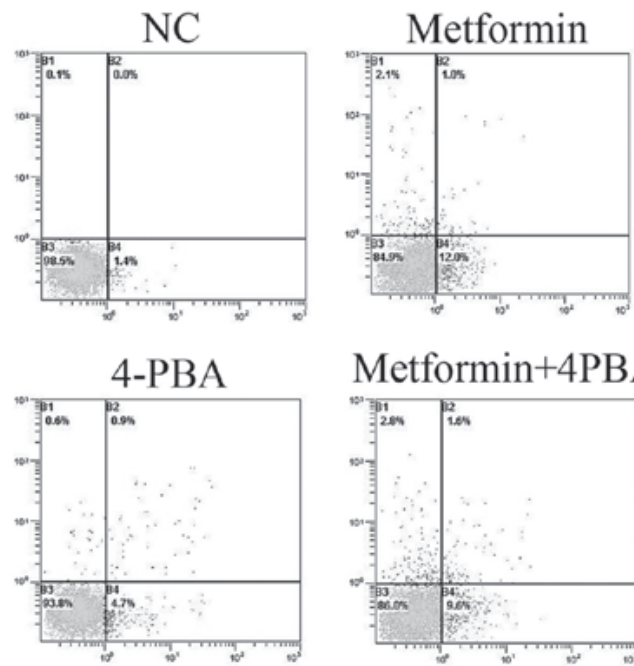

B

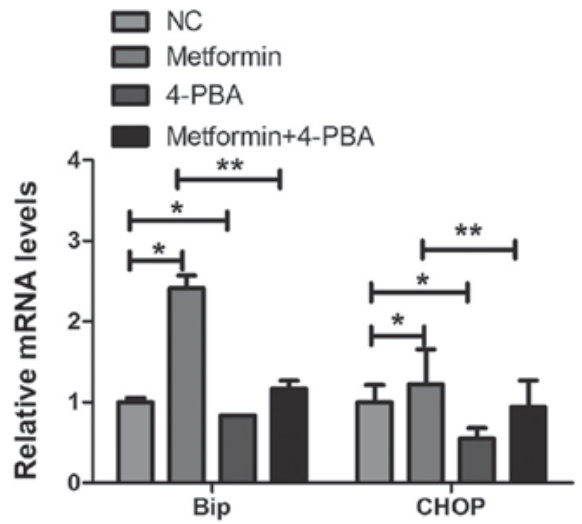

D

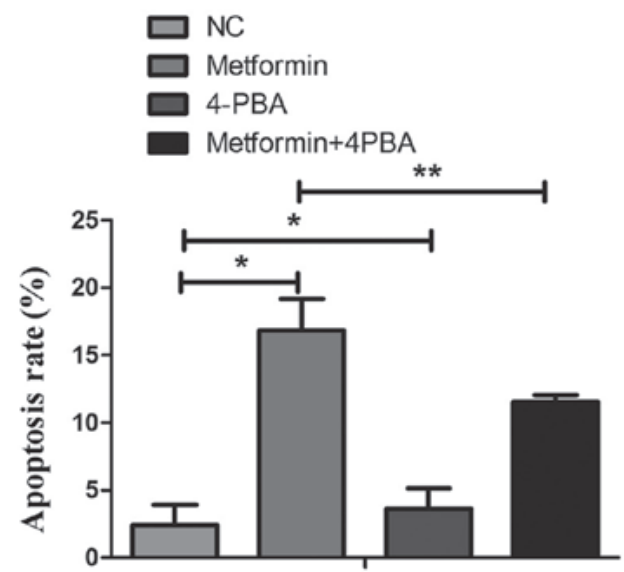

Figure 5. 4-PBA reverses the metformin-mediated apoptosis in TPC-1 cells. TPC-1 cells were pretreated with 4-PBA for 1 h, then treated with or without metformin for $24 \mathrm{~h}$. (A) The protein expression levels of Bip and CHOP were detected by western blotting, using $\beta$-actin as a loading control. (B) mRNA expression levels of Bip and CHOP following treatment with 4-PBA and/or metformin in TPC-1 cells. (C) TPC-1 cells were stained with Annexin V-FITC and propidium iodide, and analyzed flow cytometry. Representative plots are shown (D). Quantification of apoptosis rate. * $\mathrm{P}<0.05$, with comparisons indicated by brackets. 4-PBA, 4-phenylbutyrate; Bip, heat shock protein family A member 5; CHOP, DNA damage-inducible transcript 3; NC, negative control; Met, metformin.

ER-associated apoptosis genes CHOP and caspase-12, were detected in TPC-1 cells by RT-qPCR and western blotting. As presented in Fig. 3, the mRNA and protein expression levels of Bip, CHOP and caspase-12 were significantly increased with increased concentrations of metformin for $24 \mathrm{~h}$. These data suggested that metformin may induce ER stress-mediated apoptosis.

Activation of ER stress by thapsigargin enhances metforminmediated apoptosis in TPC-1 cells. To detect whether ER stress serves an important role in apoptosis mediated by metformin, TPC-1 cells were pretreated with the ER stress activator thapsigargin $(1 \mu \mathrm{mol} / \mathrm{l})$ for $1 \mathrm{~h}$, followed by treatment with metformin for $24 \mathrm{~h}$. As presented in Fig. $4 \mathrm{C}$ and D, treatment with thapsigargin enhanced the metformin-mediated apoptosis. Furthermore, treatment with thapsigargin and metformin further increased the expression levels of Bip and CHOP compared with either treatment alone (Fig. 4A and B). These data indicated that activation of ER stress enhanced the anticancer effect of metformin in TPC-1 cells.

Inhibition of ER stress by 4-PBA reverses metformin-induced apoptosis in TPC-1 cells. To further confirm whether ER stress has an important role in apoptosis induced by metformin, TPC-1 cells were pretreated with the ER stress inhibitor 4-PBA ( $1 \mathrm{mmol} / \mathrm{l}$ ) for $1 \mathrm{~h}$, followed by treatment with metformin for $24 \mathrm{~h}$. As presented in Fig. 5C and D, treatment with 4-PBA decreased the metformin-mediated growth apoptosis. Furthermore, 4-PBA and metformin co-treatment decreased the Bip and CHOP mRNA and protein expression levels, compared with metformin treatment alone. These results further confirmed that the ER stress process was required for metformin-induced apoptosis in TPC-1 cells.

Metformin inhibits TPC-1 tumor cell growth and increases the expression of Bip and CHOP in vivo. Next, the effects of metformin on TPC-1 cells were investigated in vivo using a xenograft mouse model (Fig. 6A). The results demonstrated that the tumor volume (Fig. 6B and C) and the tumor weight (Fig. 6D) in the control group at the end of the treatments were $391.0 \pm 157.6 \mathrm{~mm}^{3}$ and $1.08 \pm 0.25 \mathrm{~g}$, respectively, whereas those in the metformin group were $153.1 \pm 66.5 \mathrm{~mm}^{3}$ and $0.55 \pm 0.13 \mathrm{~g}$, respectively. These data indicated that treatment with metformin significantly decreased the tumor growth rate and tumor weight compared with the control group (Fig. 6B-D). The protein expression levels of Bip and CHOP in the tumor 
A

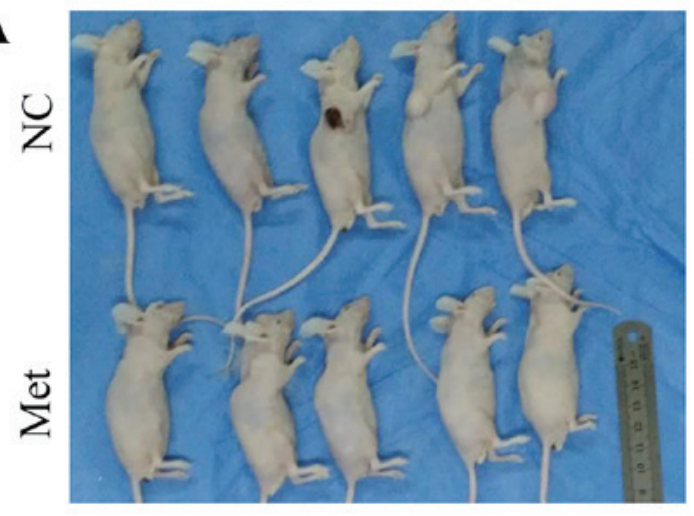

C

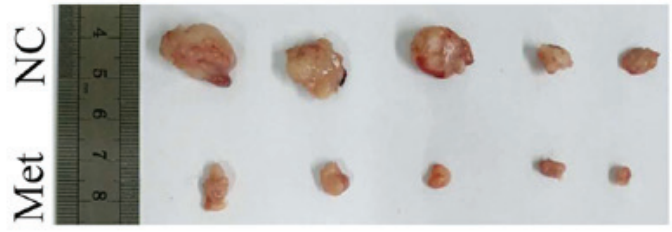

$\mathbf{E}$

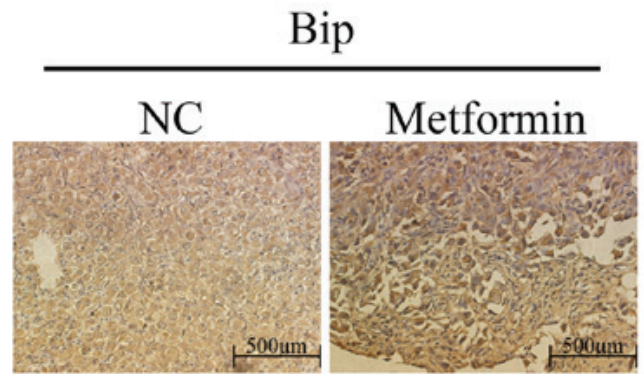

$\mathbf{F}$

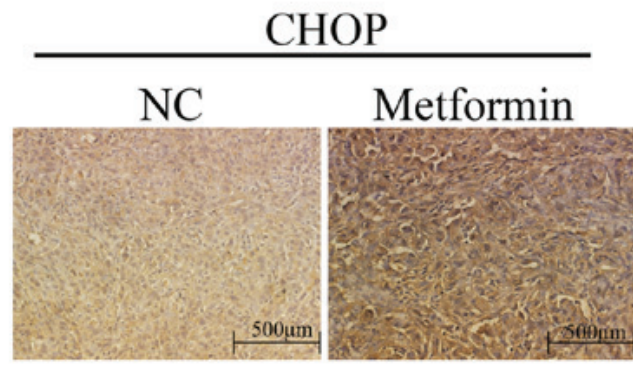

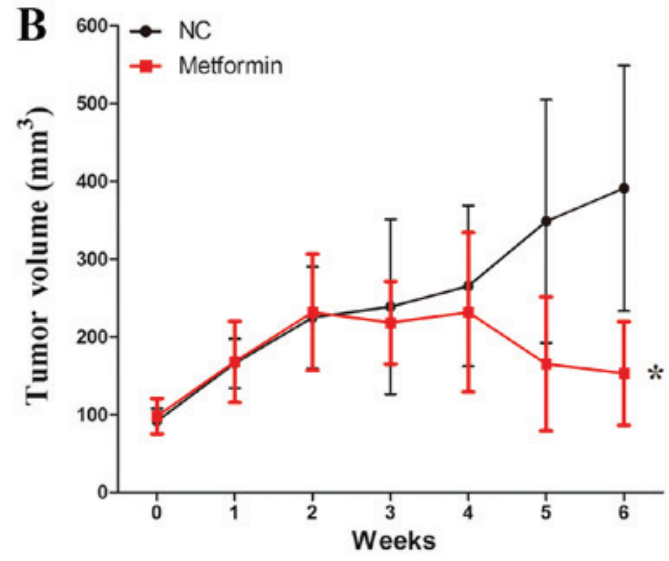

D
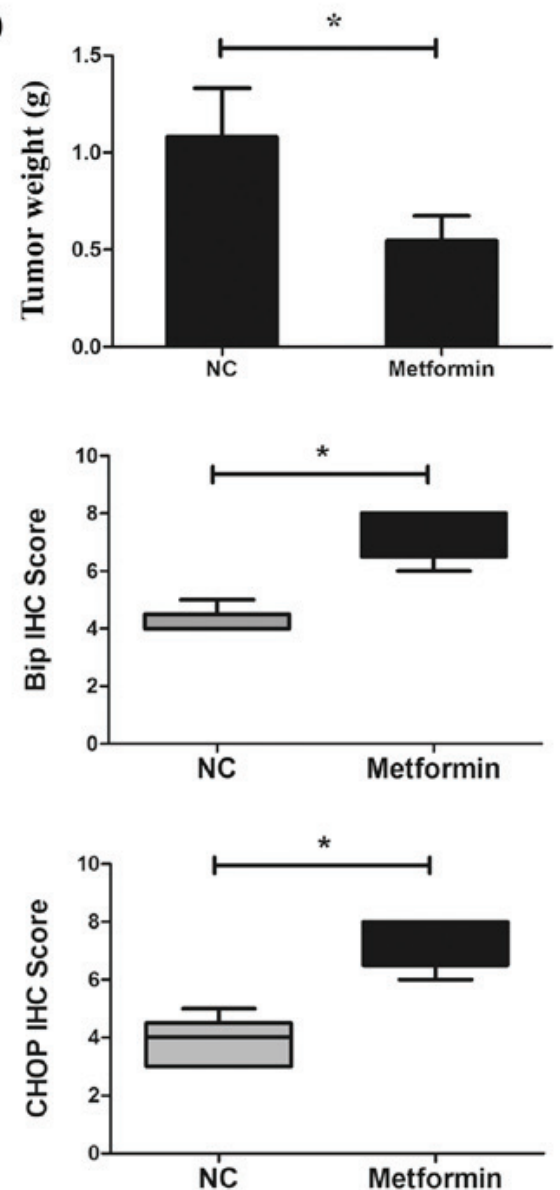

Figure 6. Metformin inhibits tumor growth in TPC-1 xenografts. (A) Tumor-bearing xenograft mice. (B) Tumor volumes over time for BALB/c nude mice following treatment with PBS or metformin. (C) Photographs of the tumors and (D) tumor weights measured in each group at the endpoint of the experiment. (E) Immunohistochemistry analysis for Bip and (F) CHOP expression in the xenograft tumor tissues. Original magnification, $\mathrm{x} 200$. "P $<0.05$. NC, negative control; Met, metformin; Bip, heat shock protein family A member 5; CHOP, DNA damage-inducible transcript 3.

xenograft tissues were detected by immunohistochemistry. As presented in Fig. 6E and F, the expression levels of Bip and CHOP were significantly increased in the tumor tissues from the metformin group compared with the control group, which is consistent with the aforementioned in vitro results of the present study.

\section{Discussion}

Metformin, commonly used for the treatment of diabetes mellitus through increasing insulin sensitivity and improving glycemic control, has been demonstrated to inhibit cancer cell proliferation and induce cancer cell death (23). Previous studies have reported that metformin inhibits the carcinogenesis of endocrine tumors through activating AMPK, leading to the downregulation of mTOR activation, which serves an important role in cell metabolism (24-26). Evidence also suggests that metformin reduces chemoresistance in ovarian cancer (27), bladder cancer (28), breast cancer (6) and esophageal squamous carcinoma (29). The present results suggested that cellular proliferation was suppressed following treatment with metformin in TPC-1 
cells. Flow cytometry analysis revealed that metformin significantly increased the rate of apoptosis in TPC-1 cells, in a concentration and time-dependent manner. Mechanistically, metformin may induce TPC-1-cell apoptosis through its regulation of ER-stress associated pathways.

In the present study, the metformin dosing range used in vitro was $1.25-20 \mathrm{mmol} / \mathrm{l}$, while the serum levels of metformin in patients with type 2 diabetes (average $1,500 \mathrm{mg}$ oral daily dose) have been reported at 2-6 $\mu \mathrm{mol} / 1$, with peak levels of $38 \mu \mathrm{mol} / 1$ and steady state ranges of $15.5 \mu \mathrm{mol} / \mathrm{l}$ (21). A recent study has indeed noted that the dosing of metformin in vitro is a contentious issue, with concerns that supra-physiological doses result in off-target effects and are not reflective of in vivo events (21). However, it can be speculated that the purpose of using metformin for diabetes treatment is the regulation of insulin sensitivity and improvement of glycemic control, while the use of metformin for cancer treatment would aim at inducing apoptosis. In the present study, the anticancer effect of metformin was explored in thyroid cancer cells, instead of a diabetic model. Our previous study also indicated that metformin at $20 \mathrm{mM}$ exhibits anticancer effects in gallbladder cancer (18). Similar in vitro concentrations have been used in other studies $(16,30,31)$.

Deregulation of apoptosis can lead to the development or progression of tumorigenesis. To investigate the mechanism of metformin-induced cell death, the ER stress pathway-mediated apoptosis was examined. Increasing evidence suggests that ER stress-associated apoptosis is involved in the apoptosis induced by anticancer agents (32). The ER triggers adaptive protective processes with the accumulation of unfolded or misfolded proteins (33). This homeostatic mechanism is mediated by UPR via three ER transmembrane receptors: Activating transcription factor 6 (ATF6), protein kinase dsRNA-like ER kinase (PERK) and inositol-requiring enzyme $1 \alpha$ (IRE1 $\alpha$ ) (34). Increasing evidence suggests that ER stress serves a protective role against tumor invasion, metastasis and chemoresistance $(35,36)$. However, when ER stress is severe or prolonged, the ATF6, PERK and IRE1 $\alpha$ signaling pathways are activated, resulting in increased expression of CHOP, caspase-12 and JNK (37), and subsequently leading to apoptosis.

Recent studies have suggested that ER stress serves a role in chemoresistance in colorectal cancer (38). A previous study has reported that ER stress induces apoptosis via the $\mathrm{JNK} / \mathrm{p} 38$ pathway following treatment with protodioscin (39). However, the role of metformin in thyroid cancer remained unclear. The present study demonstrated that the mRNA and protein expression levels of Bip increased with metformin treatment in TPC-1 cells. Bip is an abundant and key ER chaperone. It has been proposed that under conditions of ER stress, the expression of Bip is increased and the three ER stress sensors IRE1 $\alpha$, PERK and ATF6, are activated to alter transcriptional and translational programs, indicating that the expression and activation of Bip has a key role in ER stress (40). The present study also observed that the expression of the ER stress-associated apoptosis genes CHOP and caspase-12 increased when TPC-1 cells were treated with metformin. CHOP, a key transcription factor, is the most well-characterized proapoptotic pathway in the ER (41). Investigating the relationship between ER stress and apoptosis, it was demonstrated that activation of ER stress by thapsigargin enhanced the sensitivity of TPC-1 cells to metformin, while inhibition of ER stress by 4-PBA reversed metformin-induced apoptosis in TPC-1 cells. Furthermore, a xenograft mouse model was used to investigate the role of metformin in vivo. Treatment with metformin significantly decreased tumor growth compared with the control group. Furthermore, the protein expression levels of Bip and CHOP were increased in tumor tissues treated with metformin compared with the control group, which was consistent with the in vitro results. These data indicated that metformin may suppress cell proliferation via induction of ER stress-associated apoptosis. Cho et al (42) revealed that treatment with $100 \mathrm{mg} / \mathrm{ml}$ metformin decreases tumor growth by $47-60 \%$ compared with the control group in a thyroid cancer BPH10-3SC xenograft mouse model. Thakur et al (43) reported that treatment with metformin leads to a significant reduction in thyroid cancer metastatic growth in the FTC133 model, but not in the BCPAP model. A recent study also reported that metformin is a potential anticancer agent in multiple types of cancer (44). Based on these studies, metformin may possess anticancer effect in other thyroid cancer cell models. In the present study, treatment with metformin in the TPC-1 xenograft model decreased tumor growth by $\sim 60 \%$ compared with the control group. However, there are several limitations to the present results: the use of only one thyroid cancer cell line; the lack of a positive control (for example sorafenib, a known anticancer drug used to treat thyroid cancer); and the lack of other mechanistic controls to compare the effects of metformin treatment. Therefore, further studies are required in order to fully investigate the anticancer effect of metformin in ER-induced apoptosis in thyroid cancer.

Recently, Yang et al (45) reported that metformin induced ER stress-dependent apoptosis, suggesting a promising therapeutic strategy for prostate cancer. In the present study, the results demonstrated that metformin could also inhibit proliferation and induce apoptosis in thyroid cancer TPC-1 cells, by targeting the ER stress-associated apoptotic pathway. These findings indicated that metformin may have therapeutic application in the treatment of thyroid cancer. Multiple studies have recently focused on the relationship between apoptosis and autophagy. Autophagy and apoptosis are both important biological processes and their relationship is complex. Wang et al (46) revealed that autophagy activated by metformin reversed hyperglycemia-induced cardiomyocyte apoptosis in H9c2 cells. Similar results were observed in the Xiao et al and Li et al studies $(47,48)$, indicating that autophagy may have a protective role in metformin-induced apoptosis. The role and mechanism of autophagy in metformin-induced apoptosis in thyroid cancer remains unclear, and it will be investigated further in our future study.

\section{Acknowledgements}

Not applicable.

\section{Funding}

This research was supported by the National Natural Science Foundation of China (grant no. 81702863), the High School 
Key Science and Technology Project of Henan Province (grant no. 19B320039), the Outstanding Young Talent Research Fund of Zhengzhou University (grant no. 1421412090) and the Medical Science and Technology Project of Henan Province (grant no. SBGJ2018021).

\section{Availability of data and materials}

All data generated or analyzed during the study are included in this published article.

\section{Authors' contributions}

JY, LQ, KC, SS and RL performed the experiments, WZ and CZ designed the study, JY and LQ prepared and wrote the study. All authors read and approved the final manuscript and agreed to be accountable for all aspects of the work in ensuring that questions related to the accuracy or integrity of any part of the work are appropriately investigated and resolved.

\section{Ethics approval and consent to participate}

The animal protocol was approved by the Institutional Animal Care and Use Committee of the First Affiliated Hospital of Zhengzhou University.

\section{Patient consent for publication}

Not applicable.

\section{Competing interests}

The authors declare that they have no competing interests.

\section{References}

1. Xu Y, Zheng X, Qiu Y, Jia W, Wang J and Yin S: Distinct Metabolomic Profiles of Papillary Thyroid Carcinoma and Benign Thyroid Adenoma. J Proteome Res 14: 3315-3321, 2015.

2. Xing M: Molecular pathogenesis and mechanisms of thyroid cancer. Nat Rev Cancer 13: 184-199, 2013.

3. Zhou Z, Liu Y, Ma M and Chang L: Knockdown of TRIM44 inhibits the proliferation and invasion in papillary thyroid cancer cells through suppressing the $\mathrm{Wnt} / \beta$-catenin signaling pathway. Biomed Pharmacother 96: 98-103, 2017.

4. Han G, Gong H, Wang Y,GuoS andLiu K:AMPK/mTOR-mediated inhibition of survivin partly contributes to metformin-induced apoptosis in human gastric cancer cell. Cancer Biol Ther 16 77-87, 2015.

5. Feng T, Li L, Ling S, Fan N, Fang M, Zhang H, Fang X, Lan W, Hou Z, Meng Q, et al: Metformin enhances radiation response of ECa109 cells through activation of ATM and AMPK. Biomed Pharmacother 69: 260-266, 2015.

6. Qu C, Zhang W, Zheng G, Zhang Z, Yin J and He Z: Metformin reverses multidrug resistance and epithelial-mesenchymal transition (EMT) via activating AMP-activated protein kinase (AMPK) in human breast cancer cells. Mol Cell Biochem 386: 63-71, 2014.

7. Chung HH, Moon JS, Yoon JS, Lee HW and Won KC: The relationship between metformin and cancer in patients with type 2 diabetes. Diabetes Metab J 37: 125-131, 2013.

8. Hall C, Stone RL, Gehlot A, Zorn KK and Burnett AF: Use of metformin in obese women with type I endometrial cancer is associated with a reduced incidence of cancer recurrence. Int J Gynecol Cancer 26: 313-317, 2016.

9. Zhang X, Zhang X, Huang T, Geng J, Liu M and Zheng J: Combination of metformin and valproic acid synergistically induces cell cycle arrest and apoptosis in clear cell renal cell carcinoma. Int J Clin Exp Pathol 8: 2823-2828, 2015.
10. Chen X, Hu C, Zhang W, Shen Y, Wang J, Hu F and Yu P: Metformin inhibits the proliferation, metastasis, and cancer stem-like sphere formation in osteosarcoma MG63 cells in vitro. Tumour Biol 36: 9873-9883, 2015.

11. Bikas A, Jensen K, Patel A, Costello J Jr, McDaniel D, Klubo-Gwiezdzinska J, Larin O, Hoperia V, Burman KD, Boyle L, et al: Glucose-deprivation increases thyroid cancer cells sensitivity to metformin. Endocr Relat Cancer 22: 919-932, 2015.

12. Jung TW, Lee MW, Lee YJ and Kim SM: Metformin prevents endoplasmic reticulum stress-induced apoptosis through AMPKPI3K-c-Jun NH2 pathway. Biochem Biophys Res Commun 417: 147-152, 2012.

13. Sun X, Liao W, Wang J, Wang P, Gao H, Wang M, Xu C, Zhong Y and Ding Y: CSTMP induces apoptosis and mitochondrial dysfunction in human myeloma RPMI8226 cells via CHOP-dependent endoplasmic reticulum stress. Biomed Pharmacother 83: 776-784, 2016.

14. Granato M, Gilardini Montani MS, Romeo MA, Santarelli R, Gonnella R, D'Orazi G, Faggioni A and Cirone M: Metformin triggers apoptosis in PEL cells and alters bortezomib-induced Unfolded Protein Response increasing its cytotoxicity and inhibiting KSHV lytic cycle activation. Cell Signal 40: 239-247, 2017.

15. Livak KJ and Schmittgen TD: Analysis of relative gene expression data using real-time quantitative PCR and the 2(-Delta Delta C(T)) method. Methods 25: 402-408, 2001.

16. Brodowska K, Theodoropoulou S, Meyer Zu Hörste M, Paschalis EI, Takeuchi K, Scott G, Ramsey DJ, Kiernan E, Hoang M, Cichy J, et al: Effects of metformin on retinoblastoma growth in vitro and in vivo. Int J Oncol 45: 2311-2324, 2014.

17. Rico M, Baglioni M, Bondarenko M, Laluce NC, Rozados V, André N, Carré M, Scharovsky OG and Menacho Márquez M: Metformin and propranolol combination prevents cancer progression and metastasis in different breast cancer models. Oncotarget 8: 2874-2889, 2017.

18. Ye J, Chen K, Qi L, Li R, Tang H, Zhou C and Zhai W: Metformin suppresses hypoxia induced migration via the HIF $1 \alpha /$ VEGF pathway in gallbladder cancer in vitro and in vivo. Oncol Rep 40: 3501-3510, 2018.

19. He W, He S, Wang Z, Shen H, Fang W, Zhang Y, Qian W, Lin M, Yuan J, Wang J, et al: Astrocyte elevated gene-1(AEG-1) induces epithelial-mesenchymal transition in lung cancer through activating Wnt/ $\beta$-catenin signaling. BMC Cancer 15: 107, 2015.

20. Suwei D, Liang Z, Zhimin L, Ruilei L, Yingying Z, Zhen L, Chunlei G, Zhangchao L, Yuanbo X, Jinyan Y, et al: NLK functions to maintain proliferation and stemness of NSCLC and is a target of metformin. J Hematol Oncol 8: 120, 2015.

21. Davies G,Lobanova L, Dawicki W, Groot G,Gordon JR,Bowen M, Harkness T and Arnason T: Metformin inhibits the development, and promotes the resensitization, of treatment-resistant breast cancer. PLoS One 12: e0187191, 2017.

22. Xu Y, Li D, Zeng L, Wang C, Zhang L, Wang Y, Yu Y, Liu S and Li Z: Proteasome inhibitor lactacystin enhances cisplatin cytotoxicity by increasing endoplasmic reticulum stress-associated apoptosis in HeLa cells. Mol Med Rep 11: 189-195, 2015.

23. Wang J, Gao Q, Wang D, Wang Z and Hu C: Metformin inhibits growth of lung adenocarcinoma cells by inducing apoptosis via the mitochondria-mediated pathway. Oncol Lett 10: 1343-1349, 2015.

24. Reni M, Dugnani E, Cereda S, Belli C, Balzano G, Nicoletti R, Liberati D, Pasquale V, Scavini M, Maggiora P, et al: (Ir)relevance of metformin treatment in patients with metastatic pancreatic cancer: An open-label, randomized phase II trial. Clin Cancer Res 22: 1076-1085, 2016.

25. Coperchini F, Leporati P, Rotondi M and Chiovato L: Expanding the therapeutic spectrum of metformin: From diabetes to cancer. J Endocrinol Invest 38: 1047-1055, 2015.

26. Cai H, Zhang Y, Han TK, Everett RS and Thakker DR: Cation-selective transporters are critical to the AMPK-mediated antiproliferative effects of metformin in human breast cancer cells. Int J Cancer 138: 2281-2292, 2016.

27. Mert I, Chhina J, Allo G, Dai J, Seward S, Carey MS, Llaurado M, Giri S, Rattan R and Munkarah AR: Synergistic effect of MEK inhibitor and metformin combination in low grade serous ovarian cancer. Gynecol Oncol 146: 319-326, 2017.

28. Su Q, Tao T, Tang L, Deng J, Darko KO, Zhou S, Peng M, He S, Zeng Q, Chen AF, et al: Down-regulation of PKM2 enhances anticancer efficiency of THP on bladder cancer. J Cell Mol Med 22: 2774-2790, 2018. 
29. Mynhardt C, Damelin LH, Jivan R, Peres J,Prince S, Veale RB and Mavri-Damelin D: Metformin-induced alterations in nucleotide metabolism cause 5-fluorouracil resistance but gemcitabine susceptibility in oesophageal squamous cell carcinoma. J Cell Biochem 119: 1193-1203, 2018.

30. Tang JC, An R, Jiang YQ and Yang J: Effects and mechanisms of metformin on the proliferation of esophageal cancer cells in vitro and in vivo. Cancer Res Treat 49: 778-789, 2017.

31. Zhang J, Li G, Chen Y, Fang L, Guan C, Bai F, Ma M, Lyu J and Meng QH: Metformin inhibits tumorigenesis and tumor growth of breast cancer cells by upregulating miR-200c but downregulating AKT2 expression. J Cancer 8: 1849-1864, 2017.

32. Zhang J, Feng Z, Wang C, Zhou H, Liu W, Kanchana K, Dai X, Zou P, Gu J, Cai L, et al: Curcumin derivative WZ35 efficiently suppresses colon cancer progression through inducing ROS production and ER stress-dependent apoptosis. Am J Cancer Res 7: 275-288, 2017.

33. Martins AS, Alves I, Helguero L, Domingues MR and Neves BM: The Unfolded Protein Response in Homeostasis and Modulation of Mammalian Immune Cells. Int Rev Immunol 35: 457-476, 2016.

34. Hetz C, Chevet E and Harding HP: Targeting the unfolded protein response in disease. Nat Rev Drug Discov 12: 703-719, 2013

35. Wu SM, Lin WY, Shen CC, Pan HC, Keh-Bin W, Chen YC, Jan YJ, Lai DW, Tang SC, Tien HR, et al: Melatonin set out to ER stress signaling thwarts epithelial mesenchymal transition and peritoneal dissemination via calpain-mediated $\mathrm{C} / \mathrm{EBP} \beta$ and NF $x$ B cleavage. J Pineal Res 60: 142-154, 2016.

36. Fan L, Song B, Sun G, Ma T, Zhong F and Wei W: Endoplasmic reticulum stress-induced resistance to doxorubicin is reversed by paeonol treatment in human hepatocellular carcinoma cells. PLoS One 8: e62627, 2013.

37. KoraMagazi A, Wang D, Yousef B, Guerram M and Yu F: Rhein triggers apoptosis via induction of endoplasmic reticulum stress, caspase- 4 and intracellular calcium in primary human hepatic HL-7702 cells. Biochem Biophys Res Commun 473: 230-236, 2016.

38. Chen MC, Hsu HH, Chu YY, Cheng SF, Shen CY, Lin YJ, Chen RJ, Viswanadha VP, Lin YM and Huang CY: Lupeol alters ER stress-signaling pathway by downregulating ABCG2 expression to induce Oxaliplatin-resistant LoVo colorectal cancer cell apoptosis. Environ Toxicol 33: 587-593, 2018.
39. Lin CL, Lee $\mathrm{CH}$, Chen CM, Cheng CW, Chen PN, Ying TH and Hsieh YH: Protodioscin induces apoptosis through ROS-mediated endoplasmic reticulum stress via the JNK/p38 activation pathways in human cervical cancer cells. Cell Physiol Biochem 46: 322-334, 2018.

40. Wang G, Yang ZQ and Zhang K: Endoplasmic reticulum stress response in cancer: Molecular mechanism and therapeutic potential. Am J Transl Res 2: 65-74, 2010.

41. Malhi $\mathrm{H}$ and Kaufman RJ: Endoplasmic reticulum stress in liver disease. J Hepatol 54: 795-809, 2011.

42. Cho SW, Yi KH, Han SK, Sun HJ, Kim YA, Oh BC, Park YJ and Park DJ: Therapeutic potential of metformin in papillary thyroid cancer in vitro and in vivo. Mol Cell Endocrinol 393: 24-29, 2014

43. Thakur S, Daley B, Gaskins K, Vasko VV, Boufraqech M, Patel D, Sourbier C, Reece J, Cheng SY, Kebebew E, et al: Metformin targets mitochondrial glycerophosphate dehydrogenase to control rate of oxidative phosphorylation and growth of thyroid cancer in vitro and in vivo. Clin Cancer Res 24: 4030-4043, 2018

44. Morales DR and Morris AD: Metformin in cancer treatment and prevention. Annu Rev Med 66: 17-29, 2015.

45. Yang J, Wei J, Wu Y, Wang Z, Guo Y, Lee P and Li X: Metformin induces ER stress-dependent apoptosis through miR-708-5p/NNAT pathway in prostate cancer. Oncogenesis 4: e158, 2015.

46. Wang GY, Bi YG, Liu XD, Zhao Y, Han JF, Wei M and Zhang QY: Autophagy was involved in the protective effect of metformin on hyperglycemia-induced cardiomyocyte apoptosis and Connexin43 downregulation in H9c2 cells. Int J Med Sci 14: 698-704, 2017.

47. Xiao Z, Gaertner S, Morresi-Hauf A, Genzel R, Duell T, Ullrich A and Knyazev PG: Metformin triggers autophagy to attenuate drug-induced apoptosis in NSCLC cells, with minor effects on tumors of diabetic patients. Neoplasia 19: 385-395, 2017.

48. Li J, Gui Y, Ren J, Liu X, Feng Y, Zeng Z, He W, Yang J and Dai C: Metformin protects against cisplatin-induced tubular cell apoptosis and acute kidney injury via AMPK $\alpha$-regulated autophagy induction. Sci Rep 6: 23975, 2016. 